\title{
Les Big Data A L'emergence Des Data-Driven- Business-Models: Une Synthese De La Litterature
}

\section{Nabil Lazaar}

Cadre fiscal et comptable, Holding Al Omrane -Rabat

Thésard au (LSGO à l'ENCGK à l'Univérsité d'Ibno Tofail - Kénitra),

Maroc

Doi:10.19044/esj.2018.v14n28p211 URL:http://dx.doi.org/10.19044/esj.2018.v14n28p211

\begin{abstract}
This paper focuses on presenting a first step in a more in-depth literature review. In the border frame of the 'systemic' approach (Donnadieu \& Karsky, 2002), the study focused on the exploration of 'Big Data' value. It follows the process of the formation of the tax decision (Bensouda, 2009) using some properties of the new network apprehension paradigm (Penard, 2014). This is in line with many approaches of identification of the taxable substance of possession and the transmission of 'value' (Jemmar, 2010). Also, it is captured exclusively by the 'shareholder power' (Vatteville, 2008), using 'Data-Driven-Business-Models' (Hartmann et al., 2014). This business model, however, inspired their "Digital Laborer" (Fisher \& Fuchs, 2015) based on an activity whose 'positive externalities' (Collin \& Colin, 2013) go in the form of a "Cognitive Capital" (Boutang, 2007). "Big Data" is indeed the focus of the growing attention in the literature of different disciplines. In addition, it is at the heart of controversies concerning the apprehension by various "stakeholders" who are central to the development of this concept. Based on preliminary research work, this study is aimed at understanding the different dimensions of this Value in the Moroccan context. It also aims to shed light on why its various 'stakeholders' are apprehensive regarding this 'common good', which escapes real tax measures that can mitigate the 'negative externalities' of this new statistical power (Rouvroy \& Berns, 2010).
\end{abstract}

Keywords: Stakeholders, Big Data, Immaterial Capital, Data-DrivenBusiness-Model, Network externalities

Résumé

Cette communication a pour objectif de présenter une première étape d'une revue de littérature plus approfondie, s'inscrivant dans le cadre d'une approche «systémique» (Donnadieu \& Karsky, 2002), portant sur 
l'exploration de la «valeur» des «Big Data» suivant le processus de la formation de la décision fiscale (Bensouda, 2009) et usant de quelques propriétés du nouveau paradigme d'appréhension des réseaux (Penard, 2014). Ceci dans un essai d'identification de la substance taxable de la possession et la transmission de la « valeur» (Jemmar, 2010) captée exclusivement par un «Pouvoir actionnarial» (Vatteville, 2008) utilisant des «Data-DrivenBusiness-Models » (Hartmann et al., 2014), inspirants leur « Digital Laborer » (Fisher \& Fuchs, 2015) une activité dont les « externalités positives 》 (Collin $\&$ Colin, 2013) vont sous la forme d'un « Capital Cognitif» (Boutang, 2007). Ainsi, les «Big Data » font en effet, l'objet d'une attention croissante dans la littérature de différentes disciplines et se trouvent au cœur de controverses concernant leur appréhension par les différentes «parties prenantes » à leur création. L'objectif que nous nous fixons pour notre travail de recherche préalable, est de comprendre les différentes dimensions de cette "valeur» dans le contexte marocain, et pouvoir apporter des éclaircissements sur l'appréhension des différentes "parties prenantes 》 de ce "bien commun " qui échappe à de vraies mesures fiscales pouvant atténuer les "externalités négatives » de ce «nouveau pouvoir statistique » (Rouvroy \& Berns, 2010).

Mots-clés : Parties prenantes, Big Data, Capital immatériel, Data-DrivenBusiness-Model, Externalités de réseaux

\section{Introduction}

Plusieurs indicateurs poussent à croire que nous vivons dans une ère différente, dont le "Tout Numérique » et les technologies et techniques avancées d'analyse, permettent de créer de nouveaux types de matières et d'idées à partir d'une quantité volumineuse de données avec un degré de sophistication, de rapidité et de précision irréalisable jusqu'à lors, communément appelée « Big Data $(B D) »$.

De ce fait, l'économie numérique entretien une ambiguïté majeure, centralisée autour du concept de "la création de la valeur », comme décrite très tôt par Gensollen M. (1999), qui prônait déjà qu'il y a dans cette économie, un processus fondateur de transformation de la gratuité en valeur marchande, expliquant l'aspect hybride des «Business Model (BM)» et leur construction évolutive. Ceci dit, nous nous sentons forcés d'admettre, que cette ambiguïté ne cesse de croître à l'analyse des fondamentaux des (BM) de l'internet. Singularisant du coup l'économie dont ils dépendent par la dominance des " effets de réseau directs et indirects", où les consommateurs restent des agents économiques passifs subissant un nouveau mode de production des firmes inspirant de nouvelles formes du «Digital Labor», dont les «externalités 》 vont sous la forme d'un «Capital Cognitif» de Moulier Boutang Y. (2007) sans contrepartie et portant préjudice à la vie privée des 
personnes, voire poser de réelles questions d'autodétermination de ces dernières.

La littérature est passée donc, par des interrogations sur les différentes perceptions de cette valeur auprès des «parties prenantes » à la lumière des nouveaux paradigmes de l'analyse du réseau complexe (Pénard Thierry \& Rallet Alain, 2014) créée autour du «Data-Driven-BusinessModels (DDBM)».

Le flou et l'imprécision auxquels nous sommes confrontés en tentant de déterminer ces dimensions, justifiera dans une certaine mesure, notre approche «Systémique », réfutant tout réductionnisme analytique, et toute prétention d'une approche «Positiviste» à expliquer l'ensemble par ses éléments. Faisant écho à la remarquable formule de Blaise Pascal contenant en germe le fondement même de cette approche et rapportée par (Cambien, 2007): " Toutes choses étant causées et causantes, aidées et aidantes, médiates et immédiates, et toutes s'entretenant par un lien naturel et insensible qui lie les plus éloignées et les plus différentes, je tiens impossible de connaître les parties sans connaître le tout, non plus que de connaître le tout sans connaître particulièrement les parties ». En effet, la recherche de similitudes entre disciplines apparemment très éloignées par le « savant fiscaliste », se trouvent au centre de notre recherche, ayant comme ambition la collecte d'idées, de méthodes et de terminologies d'appartenant à plusieurs domaines et s'inscrivant dans un parcours caractérisé par de multiples aller-retour entre plusieurs «triangulations systémiques» interdépendantes: "Machine, Organisme et Société » ou encore entre «Bien, Valeur et Marché ».

Une des causalités circulaires que présente le « système en réseau » que nous étudions, mais qui est loin d'être la seule, car en effet, l'avènement des (DDBM) combinant les «Big Data» et les «Data Mining », créant une «boucle de rétroaction négatives » rendant difficile de distinguer entre « l'effet et la cause » de la Valorisation des données personnelles. Renvoyant au fameux paradoxe de la poule et de l'œuf relevé par Denadieu G. et Karsky M. (2002) : "l'effet rétroagit sur la cause qui devient effet et il est impossible de dire qui se trouve à l'origine! » ou en d'autres termes à une « Homéostasie » du système en question, dont le «Pouvoir actionnarial » monopolise dans une grande mesure la captation de la Valeur maintenant finalement une « dynamique stable » où le «Winner-take-all».

Paradoxalement, encore une fois, à même la stabilité dont jouit ce système, la «dynamique de changement» n'est pas en soi écartée. Au contraire, dans de tels systèmes, une fois la masse critique d'utilisateurs atteinte, les effets des «boucles de rétroaction positives » assurent la diffusion et le succès de leurs services (Varian \& Shapiro, 1998). En combinant de façon novatrice la relation entre les deux côtés du marché, par des stratégies tenants compte du phénomène de "double externalité de réseau », en s'efforçant à 
affronter les sources de destruction et d'absorption de la valeur par les acteurs extérieurs à leurs écosystèmes propres, notamment en se prêtant à l'optimisation fiscale planifiée, à la récréation perpétuelle de la valeur et enfin à l'adaptation continue des chaînes de valeur traditionnelles.

En revanche, nous ne cachons pas, que notre posture « systémique », est fortement inspirée du paradigme "interprétativiste », favorisant la compréhension de l'objet d'étude, par le sens que les acteurs donnent à la réalité, tout en admettant que la réalité ne sera jamais interdépendante de la conscience de ce lui qui l'observe. En priorisant la compréhension du sens à celui de l'explication de la fréquence, en captant le comment de la construction de celui-ci dans et par les interactions, les pratiques et les discours.

Il convient également de préciser - et à juste titre -, qu'il s'agit d'une recherche empirique, se maintenant sur le plan de l'expérience des acteurs. Ainsi, ce genre de recherche fait appel à la rétention des données, au recueil des faits ayant pour objet de répondre à une question de recherche particulière. Ceci dit, dans le cadre "interprétativiste », s'inscrit souvent dans une démarche hypothético inductive alternant déduction et induction. Cependant, cela n'empêche que l'objet de recherche peut des fois imposer l'abduction, d'où le recours à celle-ci, pour les besoins de notre recherche, et dégageant plusieurs similitudes avec la vision «Systémique » discutée ci-dessus, vu que nous ne prétendons pas définir une règle d'ensemble, mais plutôt des résultats (des mesures fiscales pour notre projet de recherche doctoral) qui vont soutenir des hypothèses préparant le terrain à des recherches futures. En fait, le chercheur n'est pas " positiviste ", " interprétativiste 》 ou " constructiviste " : il l'est par le triptyque « épistémologie, méthode, objet de recherche» (Usunier, et al., 1993).

Finalement, le mode opératoire « mixte » de notre étude empirique, sera basé à la fois sur des questionnaires quantitatifs en ligne et des entretiens semidirectifs effectués auprès de plusieurs échantillons représentatifs, ainsi qu'une étude documentaire approfondie des données secondaires de quelques entreprises numériques marocaines.

Cette approche reste motivée en grande partie par la double affection que nous portons à l'objet de recherche, en étant à la fois « fiscaliste » de profession et « Producteur de données personnelles » de conjonction !

Ceci dit et dans le cadre restreint de cet article, nous souhaitons présenter les apports de différentes théories à la construction de la Valeur des (BD). La synthèse de la littérature sur la question a pour objet de faire connaître les débats fondamentaux, nécessaires à la compréhension de la création d'une valeur propre à l'économie des données.

Nous portons l'attention, tout d'abord, sur l'appréhension de la création de valeur dans l'économie numérique suivant les apports théoriques différents. Puis dans un deuxième temps nous nous focaliserons sur quelques typologies 
recensées par la littérature des (DDBM) et leur raison d'être issue du « travail gratuit » des utilisateurs.

\section{La révolution numérique est en marche}

Une analyse profonde de l'orientation future des agents économiques et de leur perception du partage de la Valeur, requiert une connaissance succincte du terrain de l'étude - environnement dont lequel les facteurs de développement de ces orientations prennent forme. C'est ainsi que plusieurs indicateurs économiques convergent dans l'idée, que la satisfaction liée aux gains de qualité et de diversité a atteint ses limites pour un grand nombre de biens et de services, dont le régime de croissance sollicite des ressources colossales pour une utilité marginale de plus en plus disproportionnée, défavorisant au passage une part croissante de la population, et signalant par ce ralentissement des gains de productivité, l'épuisement d'un régime de croissance.

Ce constat est d'autant plus marquant à la lecture de la "quasitotalité » des rapports d'institutions économiques et de leurs sous-comités d'experts internationaux, admettant que la digitalisation est "L'évolution " radicale depuis la révolution industrielle, du fait de son abondance en opportunités d'innovation, créations d'entreprise et d'emplois, tout en constituant l'un des principaux moteurs du développement durable. Pour cela, la littérature n'a pas cessé de débattre au cours des deux dernières décennies autour du terme de "l'économie numérique», tentant d'extraire ses caractéristiques intrinsèques la distinguant de celle des autres aspects de l'économie traditionnelle.

Ainsi, d'après l'OCDE «L'économie numérique a plusieurs caractéristiques : un recours sans équivalent à des actifs incorporels, l'utilisation massive de données (et notamment de données personnelles), l'adoption généralisée de modèles d'activité à plusieurs faces qui créent de la valeur à partir d'externalités générées par des produits gratuits, et la difficulté de déterminer le pays dans lequel la valeur est créée ", une définition largement inspirée du rapport Colin-Collin, qui pointait quatre ans auparavant, la dominance de la gratuité et les progrès des technologies combinés aux données issues du «Travail Gratuit» des utilisateurs, qui ont conduit à l'émergence des «Big Data», rendant la tâche encore plus difficile pour mesurer la Valeur ajoutée issue de l'augmentation tendancielle du surplus du consommateur.

\subsection{Remise en cause de notre conception de la création et du partage de Valeur}

Du point vu de la pratique, le processus de Valorisation peut être considéré comme n'étant plus que la réponse à une seule question : "Qu'est- 
ce que la Valeur?», une question pour laquelle la théorie n'est pas restée indifférente, comme écrit Fishman, Jay E. et al. (2007), qui rappellent que la définition de la valeur passe avant tout par l'identification de la norme de Valeur, qui contient pour le coup plusieurs hypothèses représentant pour chacune d'entre elles, les fondements du type de valeur utilisé dans une convention particulière. En ajoutant que même lorsqu'une norme de valeur est spécifiée, il n'y a aucune garantie que toutes les parties prenantes de cette même convention se mettent d'accord sur les hypothèses sous-jacentes de cette norme et reprennent, pour illustrer d'avantage cette idée, les propos de Bonbright J. C. (1937), qui critiquait l'ambiguïté que contiennent les définitions de valeur conventionnelles ainsi que de l'acceptabilité de ces concepts pour certaines finalités et pas pour d'autres.

D'où, la nature polysémique du concept. En effet, chaque domaine des sciences décline le thème de la Valeur par son propre prisme, déformant ainsi, toute définition réduite satisfaisante à toute partie prenante y contribuant. Ainsi, d'un point de vue économique, et selon l'approche marginaliste ou néoclassique, par ses fondateurs Jevons W.S. (1860), Menger C. (1871), Warlas L. (1874) et Pareto V. (1895), c'est l'utilité marginale d'un bien qui détermine sa Valeur, résultat de la perception subjective des agents économiques. Selon ces auteurs, on ne cherchera plus désormais à déterminer ce qui donne de la Valeur aux choses, mais on s'intéressera plutôt aux mécanismes de fixation des prix, marquant ainsi ce qu'on appellera depuis, la "Révolution marginaliste " en contradiction totale avec l'approche objectiviste des théoriciens classiques, Smith A. (1776), Ricardo D. (1811) et dans une certaine mesure Marx K. (1867), qui prônait l'importance du travail, comme déterminant essentiel de la Valeur des choses produites.

La Valeur dont traite la littérature en finance d'entreprise quant à elle, privilégie une partie prenante Capitale, représentée par le "Pouvoir actionnarial », qui comprend au plus bas niveau de son élaboration la variation de la capitalisation boursière d'une entreprise sans incorporer ses éléments de performance liés à ses propre comportements économiques, fixant ainsi, un objectif de maximisation de la richesse de ses détenteurs et réduisant la Valeur créée à la simple rentabilité des capitaux investis - c.-à-d. "Qu'il n'y a création de valeur que si la rentabilité économique des investissements est supérieure au coût moyen pondéré du capital, fondement des critères de la Market value Added (MAV) ou de l'Economic Value Added (EVA) " et reposant sur les trois piliers identifiés par Vatteville E. (2008) pour définir la "Valeur Actionnariale»: " un fondement idéologique fort, une théorie financière sophistiquée, une comptabilité "financiarisée" »- et symbolisant une forme de «dictature de l'actionnaire » comme l'écrit Lorino P. (1998).

Ces propos viennent enrichir les apports de la théorie des parties prenantes, démystifiant l'hypothèse que tous les apporteurs de facteurs de 
production, autres que les actionnaires, sont rémunérés à hauteur du prix établi sur le marché, en postulant qu'une telle vison de la Valeur est réductrice, du fait des conséquences qu'entrainent les décisions de la firme pour l'ensemble de ce qu'ils appellent désormais les «Parties Prenantes $(P P) »$ ou «Stakeholders $(S H)$ ». Laquelle appellation, dans le sens le plus large, est proposée par Freeman R.E (1984) et reprise par Carroll A. B. et Buchholtz A. K. (2000): "Une partie prenante est un individu ou groupe d'individus qui peut affecter ou être affecté par la réalisation des objectifs organisationnels».

Par-là, ce débat de fonds, tend à reformuler la théorie de la firme dans la lignée de ce que l'on a appelé la « Révolution managériale », en proposant une vison pluraliste de celle-ci, en la représentant comme un nœud de contrat entre les différentes PP ou encore constituant un jeu coopératif entre ces dernières. L'apport de Porter M. E. (1984) à cette conception n'est pas des moindres, en vue des raisonnements fondés sur l'analyse de sa chaîne de valeur par de nombreux auteurs le succédant, afin, d'identifier une mesure de l'appropriation de la Valeur créée. Notamment, par la conceptualisation de la Valeur concurrentielle résultante du choix et de la combinaison des activités de l'entreprise, ou encore, par l'intégration de la perception des clients de celleci et le prix qui fonde la compétitivité de l'entreprise. Enfin, cette évolution des paradigmes de ladite Valeur, s'est accompagnée par un mouvement « vert», poussant les «managers» à mettre l'accent sur les dimensions environnementales, économiques et sociales au centre de leurs préoccupations.

Ainsi, depuis les textes fondateurs du concept par Dodd E. M. Jr (1932) et Barnard C.I (1938), puis de sa redécouverte par Ansoff I. (1968) et son approfondissement par Freeman R. E. (1984), la théorie des PP a fait son bout de chemin. Conduite par d'autres travaux dont l'objectif est de mieux affiner la notion, à l'instar de Frooman J. (1999) qui considère l'existence d'une interdépendance réciproque entre toutes les PP, dépassant la vision classique représentant la firme au centre du nœud de contrats avec les différentes PP, tout en recommandant l'introduction d'un aspect dynamique des PP. Une recommandation intégrée à une certaine mesure dans le modèle proposé par Mitchell Ronald K., et al. (1997), lequel modèle, reste majoritairement le plus mobilisé par la littérature où les auteurs fournissent une grille de trois (3) attributs pour hiérarchiser les (PP) : (1) «Le Pouvoir », (2) "La légitimité » et "L'urgence - lequel critère retiendra notre attention pour les besoins de notre travail $\rightarrow$. Un dynamisme du modèle des (PP) souhaité aussi par Mercier S. (2001) dans sa revue de littérature portant sur ce sujet, partant de la critique visant le cœur du fondement de la conviction des auteurs de cette théorie, en les accusant, de puiser dans les références philosophiques et métaphoriques au détriment d'une approche normative plus robuste, pour qu'elle puisse être testable. Réduisant ainsi, la création de la "Valeur Partenariale » qu'elle prône, à une simple : " contrainte limitant les objectifs 
économiques, qu'une finalité englobante, composante dominante de la politique générale ».

Cela étant dit, la littérature récente au sujet de la création et du partage de la Valeur, n'est pas restée figée à ce stade, notamment, par l'introduction de la notion de la "Création de la Valeur Partagée », qui s'inscrit dans la continuité de la Valeur dite "orientée client ou coconstruite ", qui a contribué à modifier la manière de comprendre ladite Valeur, désormais dynamique dans un modèle en interaction. Cette nouvelle notion, explorée dans un premier article par Porter M. et Karmer M. (2006) par l'établissement d'un lien étroit, entre la Responsabilité Sociale des Entreprises (RSE) et les priorités des dirigeants -, le second article en (2011), vient développer le concept au-delà de celui de la (RSE) en distinguant trois leviers qui participent à la création de la valeur partagée : (1) "concevoir autrement ses produits et marchés "; (2) "repenser sa chaîne de valeur 》; (3) "intégrer un cluster localement». Cela dit, pour de nombreux auteurs sous-estimant la portée du concept évoqué par Porter et Karmer le plaçant pas loin de celui du «Business case » pour RSE.

\subsection{Le Business Model comme lieu privilégié de la rencontre des Parties Prenantes, de la création et du partage de Valeur :}

Le débat sur la création et le partage de la Valeur ne peut être riche sans évoquer, un concept dont l'utilisation ne cesse de rencontrer une croissance exponentielle par la sphère académique depuis déjà une vingtaine d'années à savoir celui du «Business Model $(\mathrm{BM}) »$. Un concept qui vient créer une nouvelle polémique autour de lui, tant par la diversité des dimensions thématiques dont il traite, - (Jouison, 2008 ; Jouison \& Verstraete, 2009) dans la dimension entrepreneuriale (Demil \& Lecoq, 2010 ; Demil \& Lecoq, 2008 ; Lecocq et al., 2006 ; Warnier V. et al., 2004 ; Moigeon \& Lehmann-Ortega, 2010) dans la dimension stratégique - que pour la confusion que prête la multitude (1) des termes et définitions utilisés, (2) des représentations qu'on en fait, (3) et des composantes et leurs relations entre elles.

Ainsi, Osterwalder A et Pigneur Y (2010), définissent le (BM) comme étant la logique par laquelle : "Une organisation crée, offre et capture la valeur », tout en identifiant neuf segments basiques, formant leur outil «BM CANEVAS » qui décrit la façon logique par laquelle celle-ci a l'intention de faire de l'argent. Tandis que Verstraete T. et al. (2012), considèrent que le (BM) est plutôt " une convention relative à la génération de la valeur, à la rémunération de celle-ci et au partage de cette rémunération », et identifient trois composantes communes à leur outil conceptuel qui baptisent " $B M G R P$ » pour (Génération, Rémunération, Partage).

Cependant, une critique majeure est à porter au passif de telles conceptualisations du (BM), ne s'adressant pas à l'ensemble des (PP) et ne 
permettant pas l'identification de toutes les autres activités relatives à la construction des "Propositions de Valeurs", voire même, celles que l'organisation ne souhaite pas effectuer et qu'elle peut externaliser. Dans ce sens, en se basant sur la littérature des (PP), Berthelot Eric et al. (2014) identifient dix (10) principaux groupes de (PP) d'une organisation : (1) «Les Dirigeants », (2) "Le Noyau Dur, regroupant : Les Actionnaires, Les Salariés, Les Clients, Les Fournisseurs/Sous-traitants », (3) "Les Pouvoirs Public Nationaux», (4) "Les Pouvoirs Public Locaux», (5) "Les Instituts Financiers", (6) "Les Porteurs d'Image», (7) "Les Organisations Sociétales », (8) "Les Concurrents », (9) "Les Instituts internationaux » et enfin (10) "La Société Civile ». Une liste, certes dense, ne prétendant pas l'exhaustivité, comme le rappellent les auteurs eux-mêmes, en reprenant les propos de Martinet A. C. (1984) qui affirme que les frontières entre les différentes (PP) sont si minces, que toute partie prenante donnée, peut appartenir à un ou plusieurs groupes différents, "...où un salarié peut aussi être client ou actionnaire entre autres, ubiquité des parties prenantes ». Ces derniers, proposent une nouvelle catégorie des (PP) qui nomment «Parties Prenantes Fantômes », car ne pouvant pas être ou ne sont pas encore identifiées par le (BM) d'une organisation donnée.

Ainsi, comme nous l'avons vu plus tôt et par le biais du modèle de Mitchell Ronald K., et al. (1997), les (PP) peuvent influencer et s'influencer les unes les autres à hauteur du poids de chacun des critères dudit modèle, ou a Minima d'obtenir le dernier attribut «L'urgence » déterminent le degré pour lequel celles-ci sollicitent l'attention immédiate de l'organisation, mesurée par : le temps du traitement, l'importance de la demande et la relation entre l'entreprise et ces/cette partie.s prenante.s.

Ainsi, le rôle du (BM), serait non seulement un cadre « servant la cristallisation des relations entre futures $(P P)$ » comme chez Verstraete T. et Jouison E. (2007), mais dépasse celui-ci pour constituer « un outil de médiation entre les attentes des divers acteurs impliqués dans la vie d'une entreprise ». En approchant le (BM) de cette sorte, nous ressortons par une prise en compte plus large de la contribution des différentes (PP) à la création de Valeur - elle-même base de tout (BM) -, modélisé mathématiquement par une fonction proposée par Berthelot Eric et al. (2014) comme suit : " $B M=$ $S V P=f(S 1 V P, S 2 V P \ldots S n V P)$ », où les auteurs admettent, une proportion relative de proposition de valeur - d'ailleurs positive, négative ou nulle - à chaque (PP). De ce fait, la quantification et la rémunération de cette valeur reposera sur des critères extra-financières de bien-être et de satisfaction personnelle future des (PP), générant à travers ces (BM) une forte asymétrie sur la finalité même de la rémunération de la Valeur créée, le réduisant au passage qu'au simple reflet d'une notion très subjective de cette même Valeur. 


\subsection{L'appréhension de la création de valeur dans l'économie numérique par la dynamique qui lui est propre}

L'économie numérique entretien une ambiguïté majeure, centralisée autour du concept de "la création de la valeur », comme décrite très tôt, par Gensollen M. (1999), qui prônait déjà, qu'il y a dans cette économie, un processus fondateur de transformation de la gratuité en valeur marchande, expliquant l'aspect hybride des (BM) et leur construction évolutive. Or, le concept même de $(\mathrm{BM})$ ne demeure pas non plus exempté de tout débat académique, et dépasse de loin le cadre de son économie d'apparition et par conséquent sa définition évolue selon la posture épistémologique de chacun des chercheurs qui se sont emparés du sujet, mais gardent néanmoins comme dénominateur commun la notion de "Valeur».

Cela dit, nous nous sentons forcés d'admettre, que cette ambiguïté ne cesse de croître à l'analyse des fondamentaux des (BM) de l'internet, singularisant du coup l'économie dont ils dépendent par la dominance des " effets de réseau directs et indirects ». Un concept largement évoqué par la littérature, tant du côté de la demande que celui de l'offre, on citera à juste titre, les « externalités de réseaux » selon Katz et Shapiro (1985), les "rendements croissants d'adoption" par Arthur W. B. (1989), "l'approfondissement épistémologique » du concept par Liebowitz, S. et Margolis, S. (1994), et plus spécialement la « loi » de Metcalfe R. (1980), formulée par Gilder G. (1993) selon laquelle "la valeur d'un réseau serait égale au carré du nombre de ses usagers ", dont est sous-estimée la teneur notamment par, Odlyzko A. et Benjamin T. (2005), en postulant que « la valeur d'un réseau de taille (n) crô̂t comme nlog (n)».

Dans ce cadre, les (BM) du numérique combinent de façon novatrice la relation entre les deux côtés du marché, par des stratégies tenant compte du phénomène de "double externalité de réseau », en s'efforçant à affronter les sources de destruction et d'absorption de la Valeur par les acteurs extérieurs à leurs écosystèmes propres, notamment en se prêtant à l'optimisation fiscale planifiée, à la récréation perpétuelle de la valeur et enfin à l'adaptation continue des chaînes de valeur traditionnelles.

Prendre ainsi le (BM) du numérique pour l'un des composant de l'objet de recherche, revient aussi à s'interroger sur ses typologies basées sur les frontières de l'entreprise, sa structure de coûts et les relations qu'elle entretient avec ses partenaires et concurrents. De ce fait, en s'appuyant sur les travaux de Mckelvey M. (2001), les auteurs Brousseau E. et Penard T. (2007) proposent un cadre d'analyse des $(\mathrm{BM})$ du numérique, où ils observent au passage la nature modulaire des activités qui en découlent, en disséquant ces activités en trois opérations basiques :

"(Production, Assemblage et Consommation) », dont "les utilisateurs (consommateurs) » ne sont pas neutres vis-à-vis du processus de création de 
valeur, et enfin suggèrent trois dimensions pour benchmarker la plupart des (BM) du numérique relevant de trois domaines contradictoires de la littérature à ce sujet, à savoir : "l'économie de l'intermédiation », "l'économie de l'assemblage » et "l'économie de la gestion de connaissance », sans omettre de préciser qu'en pratique, les trois dimensions s'intègrent à des proportions inégales.

\section{La révolution des « Big Data » est en course de pointe}

\subsection{Une nouvelle ruée vers les Business Model Pilotés par les Big Data}

Un (BM) classique quand bien même, il soit couronné de succès, en captant de la Valeur latente des progrès technologiques, il n'en demeure pas moins contraignant pour la recherche de modèles alternatifs, accueillants des technologies plus poussées à l'avenir. Ce qui laisse à penser que créer de la Valeur en tirant partie de la technologie ne serait pas une question d'incertitude technique mais aussi, une incertitude relevant du domaine social, par l'interaction des entreprises avec leurs environnements Chesbrough, H., Rosenbloom, R. (2002). Ce qui encourage de plus en plus d'entreprise à repenser leurs $(\mathrm{BM})$ déjà en place ou à en créer, autour de ce concept, vers ce qu'on appellera désormais les Data-Driven Business Model (DDBM). Requérant plus de souplesse et de flexibilité que les précédents modèles, tout en considérant les données comme un "actif stratégique " tant pour l'entreprise elle-même que pour ses partenaires.

S'appuyant sur les résultats des entretiens menés auprès de 28 chefs d'entreprises en RU et aux EU, Bulger M. et al. (2014) ont observé trois types de DDBM, distinguant celui des :

(1) «Utilisateurs de données », ayant comme préoccupation centrale l'utilisation en interne des données, soit en prise de décisions ou comme intrant dans d'autres produits et services, celui des (2) «Fournisseurs de données », jouant pour sa part, un rôle de courtage, en fournissant l'accès à d'autres agents économiques des données récoltées pour un autre usage à l'origine, et enfin celui des (3) «Facilitateurs de données», venant en support des agents en manque d'expertise, en fournissant des infrastructures externalisées et du conseil.

Pour leurs parts, Hartmann P. M. et al (2014) et suite à leur profonde revue de la littérature sur le sujet, ces auteurs ont identifiés six (6) dimensions communes pour tout (BM), à savoir :

(1) les «Ressources clé » par définition donc les DDBM ont comme ressource primordiale les données, l'intérêt à ce stade serait de connaitre leurs sources puis leurs types, (2) les «Activités clé », dispensée d'une description basée sur la chaine de valeur de Porter M. (1985) et en opposition au paradigme de programmation «Orienté-Objet », la littérature s'est plutôt positionnée en faveur de 1' « Orienté-Service » notamment par les Structures orientées service 
« $S O A »$, le développement d'interface de programmation d'application «API» avec une forte inspiration des « sciences de la persuasion », jouant un rôle prédominant dans l'expérience client. (3) la «Proposition de Valeur / Offre » qui stipule que la valeur crée pour les clients à travers l'offre est mesurée par l'expérience client reçue. (4) Le «Segment Client » qui s’appuie sur la dichotomie classique «B2B ou B2C ». (5) Le « Modèle de revenu », dans ce contexte, Rocheland F. et Cecere G. (2012), affirment que les revenus sont de plus en plus détachés des investissements au détriment de la Valeur que leur attribuent les utilisateurs captés par les opérateurs dans des revenus récurrents. Enfin, (6) La «Structure de coûts», qui suit dans une certaine mesure l'application d'une stratégie d' «Océan Bleu» au (BM) en renforçant les externalités de réseaux et profitant de l'avantage du coût spécifique procuré sur l'un des côtés du marché aux dépends de l'autre face.

Suite à quoi et après utilisation d'une analyse en grappes, les auteurs ont construit une typologie plus exhaustive des (BM) ayant comme ressource clé «Les données » dans le monde des start-up, en identifiant six types de DDBM codés de (A à $\mathrm{F})$, représentés dans une matrice $(3 \mathrm{X} 3)$ et déterminés par deux dimensions dont trois attributs chacune; Mesurant l'appréhension des modèles suivant la « Source des données »- «Données gratuites », « Données fournies par les clients» ou «Données suivies et générées 》 - et suivant les «Activités clé » - «Agrégation des données », "Analyse des données» ou « Génération des données ») -

\subsection{Les Big Data issues du « Travail gratuit » des utilisateurs sont au cour de la création de valeur}

Par ce qui précède, un dépassement de plus en plus marquant de la théorie de la firme - comme évoqué ci-dessus - se définit, mobilisant au passage une «Grande Théorie » en stratégie : sous le nom de «Ressource Based» en opposition à celle des «Droits de propriétés ». Une théorie dont les adeptes, cherchent en première mesure, l'identification des «Actifs Stratégiques », dont la «Connaissance » et les «Compétences » sont les principaux fondamentaux. Ainsi, un regain d'intérêt s'est porté à la notion du « Capital Intellectuel » étroitement liée à celle du « Goodwill », dont témoigne les nombreuses publications sur le sujet et convergeant vers l'idée que sa valeur pour les organisations est telle, qu'elle mériterait d'être mesurée. Or, la notion du «Capital Intellectuel», reste largement confondue à celle du «Capital intangible », d' "Actif intangible» et de «Capital de connaissance », en paraissant traduire des concepts similaires, pour ce, un essai de définition proposé par l'OCDE reste largement admis par la littérature, selon lequel le capital intellectuel est "la valeur économique de deux catégories d'actifs immatériels («intangible assets») : le capital organisationnel (structurel) et le capital humain.». Notons qu'à ce sujet 
Edvinsson L. et Malone M. (1999), l'uns des pionniers concepteurs relatif au capital intellectuel, suggèrent une déclinaison de celui-ci en trois composantes: "Capital Client», "Capital Humain» et "Capital organisationnel».

Ceci dit, et vu la vision du (BM) combinant de façon innombrable les externalités générées par l'économie numérique, une nouvelle révolution s'ajoute au palmarès de cette économie, à savoir celle de la «Révolution $d u$ Travail » dans une économie désormais dominée par un nouveau mode de production des firmes, "consistant à produire une application inspirant à ses utilisateurs une activité, dont les externalités positives vont, sous la forme de données, s'incorporer à la chaine de production sans contrepartie monétaire». A ce propos, Harribey J. M (2010) reprend l'apologie de «l'abeille», théorisée par Moulier Boutang Yann (2010), pour illustrer le processus «polinisateur» du Capital intellectuel dans le système productif. Brouillant ainsi la frontière entre production et consommation, ou à moindre titre, stimulant le développement de nouvelles formes de production à l'instar de la «Co-Création», l' «Unsourcing», le «Widsom of crowds», le "Crowdsourcing », le « Content Curation » et bien d'autres termes barbares, mais renvoyant vers un seul concept fondamental : celui du «Digital Labor» ou « Travail Gratuit $(\mathrm{TG}) »$.

D'après Casilli A. (2015) le (TG) serait: "La réduction de nos (Liaisons Numériques) à un moment du rapport de production ", car les activités assimilables au travail seraientt détecter comme telle, " parce qu'elles sont productrices de valeur, faisant l'objet d'un quelconque encadrement contractuel et soumises à des métriques de performance ». Une définition que l'auteur enrichi, par l'assimilation des différentes manifestations du « Micro Travail » que les (BM) du numérique engendrent, comme : «Une contribution à faible intensité et à faible expertise mise à profit via des algorithmes et des fouilles de données ». Ceci dit, l'exploitation des données numériques des utilisateurs d'internet, n'est plus restreinte aux seules opérations effectuées sur un ordinateur de bureau, mais la dépasse, en enrôlant toutes sortes d'objets connectés afin de collecter constamment des données. Un enchevêtrement tel, que les sciences sociales se sont empressées de vouloir tracer une ligne de démarcation à la fois, du côté de "l'utilisateur/travailleur », en fournissant des définitions originales d'activités mixtes "travail-loisir», - les notions de «playboor» et «weisure » condensant les mots «Play» et «Laybor» puis «Work» et «Leisure »-, et de l'autre côté des «Non-Humains» - «La robotique » et «les objets connectés 》-.

Enfin, l'auteur précise une dernière fois sa définition du (TG), en la rapprochant de celle de la «Cybertariat » proposée antérieurement par Haws U. (2003), ainsi que du «Capital cognitif» de Moulier Boutang Y. (2007), pour advenir ainsi : "Le digital Labor est un travail éminemment cognitif qui 
se manifeste à travers une activité informelle, capturée et appropriée dans un contexte marchand en s'appuyant sur des tâches médiatisées par des dispositifs numériques ». Une définition longue certes, elle nous permet d'appréhender cette notion dans sa globalité, relevant de l'interaction avec les principes mêmes qui régissent le cadre de l'économie numérique et à juste titre de l'économie des données.

Il y a là donc, une critique fondamentale à la théorie des «Ressource based », - comme évoquée plutôt -, qui postule que, pour qu'un "Actif stratégique » soit identifié ainsi, il doit présenter les caractéristiques «VRIN (Valeur, rareté, Inimitabilité, non substituabilité». Or, l'idiologie de la « rareté » fait défaut chez de nombreuses firmes adoptants des nouveaux (BM) performants, dont la valeur créée à partir de ces «Actifs stratégiques » n'est pas forcément captée par la firme, mais suivant le pouvoir de négociation des (PP) établi par ces (BM). Un constat encore plus remarquable, à la lecture de Harribey J. M. (2017) qui s'interroge sur l'origine des profits colossaux réalisés par des firmes telles que : les GAFAM, et les NATU. Il avance deux réponses possibles: (1) les utilisateurs et usagers de ces firmes sont des créateurs de valeur ; (2) Il s'agit d'une captation de rente de rareté - Voire une "Rente cognitive»-, suite au phénomène du «Value Shift », dont l'instauration de nouveaux droits de propriété via des modes d' " enclosures » sur les données et les connaissances, accroît la concentration de la valeur entre peu de mains.

De ce fait, plusieurs indicateurs poussent à croire que nous vivons dans un ère différente dont le "Tout Numérique » et les technologies et techniques avancées d'analyse, permettent de créer de nouveaux types de matières et d'idées à partir d'une quantité volumineuse de données avec un degré de sophistication, de rapidité et de précision irréalisable jusqu'à lors, communément appelée «Big Data (BD)». Brillamment définie par Nagle, T. \& Sammon, D. (2014): " data that pushes the limits of common technology available at that time ", dont les bénéfices économiques ne sont plus à prouver, vu l'abondance d'études académiques, institutionnelles et industrielles sur le sujet, louant les gains de productivité, d'avantage compétitif et d'efficacité potentielle que la détention d'un tel actif procur, et dont même les gouvernements des pays développés ou en développement s'engageaient à fournir l'accès de plus en plus via les projets « open data ».

Ceci-dit, la définition de Nagle, T. et Sammon, D. (2014), même que remarquable, restera néanmoins poétique et peu exhaustive. Or, la littérature pour sa grande part, reste imprégnée par « The definition » en trois dimensions proposées par le cabinet de conseil en technologie (Gartner, 2012). Caractérisée par les $3 \mathrm{~V}$, (1) Le «Volume », vu la baisse sans précédent des coûts de stockage du Gigabyte comme formulé par Komorowski M. (2009), (2) la «Variété », en vue de la diversité des données brutes de tous types 
stockées auparavant, (3) La «Vitesse », en vue de la vélocité des données créées et dont la rapidité d'intégration au processus de décision augmente. A ces trois dimensions, un caractère initialement introduit par (Schroeck M, et al., 2012) regain en popularité, (4) la «Véracité », qui renvoie à la fiabilité des données collectées.

En revanche, le contexte des (BD), offre le choix d'un traitement qui dépasse les seuls aspects techniques, vers d'autres sociétaux, vu l'interdépendance labyrinthique que cette notion apporte aux enjeux sémiotiques, épistémologiques, éthiques et politiques, que 1'on compare des fois à de 1' «Uranium ». Ainsi, pour chaque dimension de la définition technique sus-énoncée, l'on pourra trouver, une base non-indéniable au caractère personnel des données collectées opérant comme des «purs signaux » alors même qu'elles soient «anonymes », voire qu'elles soient individuellement très peu informatives.

En effet, par le biais d« externalités de réseaux», évoqué plutôt, la valeur potentielle de chaque donnée tend à croître en fonction du «Volume » des autres données avec lesquelles est susceptible d'être associés. Réduisant les individus qui ont participé à la création de cette valeur à des simples " émetteurs » de données temporaires, ne percevant pas sa teneur. Tant et si bien même, qu'ils puissent accéder à leurs données personnelles, leurs rapports de force seraient toujours à la faveur des responsables de traitement de cellesci, comme décrit par Andrejevic Mark (2014): "les formes de (savoir) associées aux (BD) ne sont disponibles que pour ceux qui ont accès aux machines ", lequel illustre d'avantage son propos en citant Weinberger, D. (2011), «A l'ère des Big Data la personne la plus intelligente dans la pièce est la pièce ».

La «Variété » pour sa part, renvoie à deux représentations des données «Hard » et «Soft », selon si elles sont structurées ou pas, déterminées et connues à l'avance ou pas, provenant d'individus «Humains » ou pas, à l'instar de l'utilisations de technologies d'identification par radiofréquence «RFID». Concrétisant l'enjeu social que porte les (BD), qui même au-delà d'une quelconque vision éthique et restreinte de la protection des données à caractère personnel, dégage une problématique du désintéressement de plus en plus d'individus pour la maitrise des processus de «Datafication » qu'ils subissent, en grande partie dû, au coût que représenterait leur renoncement à la contrepartie désormais difficile à dissocier de toute les manifestations de la numérisation du «Monde ».

Enfin, si un concept tel que le «Delivery before Order Making » ou «Livraison avant prise de commande » existe, c'est foncièrement à cause de la «Vitesse » de l'intégration des données collectées, mettant à rude épreuve "les processus de perception, d'entendements humains et d'énonciation des motivations ». Car en effet, à la lecture des propos d'Eric Schmidt (2010), "the 
technology will be so good it will be very hard for cpeople to watch or consume something that has not in some sense been tailored for them", nous constatons que Google comme toute autre exploitant de (BD), s'efforce d'apprendre avec d'avantage de "Vitesse" sur ses utilisateurs afin de passer de ce qui est exprimé manintenant à ce qui est voulu après, ou comme dit Eric Schmidt : " As you go from the search box [to the next phase of Google], you really want to go from syntax to semantics, from what you typed to what you meant'. C'est ainsi que d'après Rouvroy A. (2017) "l'anticipation performative » des intentions d'achat et «l'optimisation de la force de travail », posent de réelles questions d'autodétermination des personnes voire d'égalité et de non-discrimination de celles-ci.

Narayanan A. et Shmatikov V. (2010) observent ainsi qu'il devient de plus en plus difficile de faire la différence entre «les informations d'identification personnelle (IIP) 》 et «les informations non-IIP», et que toute prétention contraire est similaire au mythe médiéval des alchimistes convaincus qu'une «Pierre philosophale » pouvait transformer le plomb en or et ajoutent que «l'anonymat est devenu algorithmiquement impossible ». En effet d'après Pican X. (2015) qui reprend les propos de Grumbuch Stéphane (2012) lors de la 4ème Conférence de Lift France : « $70 \%$ des données sont produites directement ou indirectement par des personnes, consciemment ou non, Dès lors, les données de Big Data intégreront nécessairement des données à caractère personnel ». Ce qui a poussé Rouvroy A. (2017) à penser que «la gouvernance par les données » serait pour les exploitants des (BD) une « gouvernance objective » qui contiendrait toutes les informations nécessaires à une interprétation quelconque du monde indépendamment de toute perspective idéologique, poussant donc les individus à l'acceptation ou à la tolérance de la numérisation de leur environnement.

Par ce qui précède, nous sommes intéressés «délibérément», à un rapprochement de la classification des données personnelles par les attributs intrinsèques de la notion des (BD), en admettant qu'un emboitement d'une quelconque taxonomie des données personnelles est opéré de fait, en la percevant tout au long de leur cycle de vie sur les différents (BM) déployés pour y générer et y extraire de la valeur. Car après tout, les organisations axées sur les données ne se posent pas en premier lieu la question de «Que pensonsnous? » mais plutôt «Que savons-nous? ».

\section{Conclusion}

Dans cette communication nous avons tenté de prendre la mesure d'une situation nouvelle pour le cadrage théorique des «Big Data » et ce afin de dégager des perspectives de recherche. Dans un monde de données où plusieurs concepts sont taxés de polysémiques et flous, il importe de saisir des apports théoriques réfléchis dans le temps. 
En effet l'émergence des nouveaux modèles d'affaires centrés autour des données personnelles a inspirer de nouvelles formes du «travail gratuit », où les riches apports de deux cadres de réflexions dont la théorie des parties prenantes et des ressources, permettent d'y appréhender le partage de la Valeur créée. Sans omettre, et à juste titre, les contributions des auteurs conceptualisant des typologies de ces nouveaux modèles d'affaires, permettant une cristallisation des relations entre différents intervenants de la chaine des valeurs.

Dans cet esprit, l'apport des sciences sociales, n'est pas des moindre, ouvrant la voie à des approches des «Big Data », qui dépassent les seuls aspects techniques, vers d'autres sociétaux, vu l'interdépendance labyrinthique que cette notion apporte aux enjeux sémiotiques, épistémologiques, éthiques et politiques à la fois.

Enfin s'interroger sur les mesures fiscales appropriées au contexte marocain, renvoie à puiser dans plusieurs travaux descriptifs, analytiques et synthétiques (Bernard, 2002; Bensouda, 2009; Jemmar, 2010 ; Collin \& Colin, 2013; Rapports de 1'OCDE 2015a, 2015b, 2017 ; etc) -, pouvant atténuer les " externalités négatives » issues de l'exploitation de ces données.

Ceci étant dit, nous poursuivrons l'objectif ultime de soumettre $\underline{\mathbf{s a}}$ matière fiscale à un éclairage transdisciplinaire mobilisant un corpus théorique florissant et dense, tout en cherchant à mettre en évidence la nécessité d'un new public management fort par ses mesures de régulation, dont la richesse des littératures nous permettra de recueillir de façon la plus exhaustive que possible, l'ensemble des dimensions de la Valeur citées par ces dernières et qui impactera à différents degrés les mesures fiscales futures.

Nécessitant pour le coup un recensement des méthodologies d'estimation de la Valeur des «Big Data», distinguant celles se basant sur la Valeur marchande, de celles se basant sur la Valeur subjective puis celles construites économétriquement, afin d'ouvrir la voie à des recherches préalables adaptées aux pays en voies de développement plus généralement et au contexte marocain plus particulièrement.

\section{References:}

1. Andrejevic M. (2014), “The Big Data Divide", International Journal of Communication, Big Data - Big questions, n.8, pp-1676

2. Ansoff I. (1968), «Stratégie du développement de l'entreprise», éditions Hommes et Techniques, traduction française, Paris, 1968. pp41

3. Arvind N. et Vitaly S. (2010), «Privacy and security Myths and fallacies of "Personally identifiable information," Communications of the ACM, Vol. 53 (6), pp-24 
4. Aurore Cambien (2007), «Une introduction à l'approche systémique Appréhender la complexité », Rapport commandité par Centre d'études sur les réseaux, les transports, l'urbanisme et les constructions publiques, $\mathrm{n}^{\circ} 13029$, pp-13

5. Barnard, Chester. I., (1938), «The Functions of the Executive», Harvard University Press, Cambridge, 1966 pp-82.

6. Barneto P. (2012) « Mesurer la rémunération de la valeur dans le business model », Entreprendre \& Innover 2012/1 ( $\left.{ }^{\circ} 13\right)$, p. 38-47.

7. Barney J. B. (1991) "Firm Resources and Sustained Competitive Advantage", Journal of Management, vol. 17, $\mathbf{n}^{\circ}$ 1, p. 99-120

8. Barney R. (1996), "The Ressource-Based theory of the firm Organization Science, vol.7, ${ }^{\circ} 5$, septembre-octobre".

9. Bastin G. et Francony J-M (2016), «L'inscription, le masque et la donnée: Datafication du web et conflits d'interprétation autour des données dans un laboratoire invisible des sciences sociales. » Revue d'anthropologie des connaissances, vol. 10, 4,(4), pp-507.

10. Bauwens M. et Niaros V. (2016), "Value in the Commons Economy: Developments in Open and Contributory Value Accounting", pp-8

11. Ben Larbi S. et Ohanessian R. (2008) «Politiques RSE et création de valeur : Vers un modèle de gouvernance responsable ?» Archives CONGRES ADERSE 2008, L'entreprise à l'aune de ses responsabilités vis-à-vis de l'homme, de l'environnement et du profit ?

12. Benhamou F. (2013), L'irrésistible ascension de l'internet, Cahiers français, janvier-février $2013 \mathrm{~N}^{\circ} 372$

13. Béraud A. (2005), «La notion de loi dans l'oeuvre de Pareto ». Economia - History / Methodology / Philosophy, NecPlus /Association Economia, 2007, pp.1695-1017

14. Berthelot Eric \& al. (2014), «Les parties prenantes au cœur du business model ». pp-4]

15. Bessieux-Ollier C. et al. (2006) «Capital humain : mesure, management et reporting Un état des lieux sur le plan théorique et pratique.» COMPTABILITE, CONTROLE, AUDIT ET INSTITUTION(S), May 2006, Tunisie. pp-4.

16. Bonbright J.C. (1937) Valuation of Property, Charlottesville, VA: Michie Company, at 11.

17. Bouafia S. et Jaussaud, J. (2018). Projets Big Data des entreprises : quelles transformations organisationnelles? . Revue COSSI, $\mathrm{n}^{\circ} 1$ 2018

18. Brousseau E. et Penard T. (2007), The Economics of Digital Business Models: A Framework for Analyzing the Economics of Platforms, Review of Network Economics Vol.6, Issue 2 - June 2007 
19. Brulhart F. et al., (2010), " Théorie des ressources. Débats théoriques et applicabilités ", Revue française de gestion 2010/5 ( ${ }^{\circ}$ 204), pp-83.

20. Brynjolfsson E. et McAfee A, (2014), The Second Machine Age : Work, Progress, and Prosperety in a time of brilliant Technologies W. W. Norton \& Company, Inc

21. Bulger M. et al., (2014) Data-Driven Business Models: Challenges and Opportunities of Big Data, Oxford Internet Institute September 2014

22. Carroll Archie. B. et Buchholtz Ann K., (2008) "Business and Society: Ethics and Stakeholder Management», South-Western Publishing, 7ème edition, pp-84.

23. Casilli A. (2015), «Digital Labor: travail, technologies et conflictualités. Qu'est-ce que le digital labor? », Editions de l'INA, pp-4.

24. Charreaux G. et Desbrieres P. (1998) « Gouvernance des entreprises : valeur partenariale contre valeur actionnariale", Finance Contrôle Stratégie - Volume 1, N 2, juin 1998, pp-60

25. Chesbrough, H.; Rosenbloom, R. (2002), "The role of the business model in capturing value from innovation: Evidence from Xerox Corporation's technology spin-off companies", Industrial and Corporate Change, Vol. 11, No. 3, pp. 529-555.

26. Cobbaut R. (1997), « Théorie Financière », Economica 2ème éd. pp285

27. Colin N. et al, (2015), « Économie numérique », Notes du conseil d'analyse économique 2015/7 (n²6), p. 1-12.

28. Collin P. et Colin N. (2013), Mission d'expertise sur la fiscalité de l'économie numérique, Rapport au Ministre de l'économie et des finances, au Ministre du redressement productif, au Ministre délégué, chargée des petites et moyennes entreprises, de l'innovation et de l'économie numérique

29. Cook P. J., Frank R. H. (1995), “The Winner-Take-All” Society, New York: Martin Kessler Books, Free Press.

30. Dalton Conley (2009), "Elsewhere, U.S.A.: How We Got from the Company Man, Family Dinners, and the Affluent Society to the Home Office", BlackBerry Moms, and Economic Anxiety, New York, Knopf Doubleday Publishing Group

31. Debonneuil M. (2010) L'Économie quaternaire, une croissance durable à construire, Rapport de, inspectrice générale des finances, professeur à HEC et membre du Conseil d'Analyse Économique remis le jeudi 14 janvier 10 à Nathalie Kosciusko-Morizet, secrétaire d'Etat 
chargée de la Prospective et du développement de l'économie numérique

32. Desmarteau A.H., Saives A.-L., (2008) «Opérationnaliser une définition systémique et dynamique du concept de modèle d'affaires : cas des entreprises de biotechnologie au Québec ", XVIIe Conférence de l'AIMS, Nice-Sofia-Antipolis, 2008

33. Dodd, E. Merrick, Jr. (1932), "For Whom Are Corporate Managers Trustees?", Harvard Law Review, Vol. 45, No. 7 (May, 1932), pp1149

34. Donnadieu, G. \& Karsky, M. (2002). Ibd pp-25

35. Donnadieu, G. \& Karsky, M. (2002). La systémique, penser et agir dans la complexité. Paris : Editions de liaisons.

36. Eric Brousseau et Thierry Penard, (2007), The Economics of Digital Business Models: A Framework for Analyzing the Economics of Platforms, Review of Network Economics Vol.6, Issue 2 - June 2007

37. Fishman, Jay E. et al. (2007), Standards of value : theory and applications, John Wiley \& Sons, Inc.

38. Florence Allard-Poesi et Véronique Perret (2014), «Fondements épistémologiques de la recherche ", in Thiétart, R-A et coll. «Méthodes de recherche en management », 4eédition. Paris : Dunod, pp-17

39. Fogg B. J. (2002) Persuasive technology: using computers to change what we think and do, chapter-5, Magazine, Ubiquity, Volume 2002 Issue December, 2002, Article No. 5

40. Françoise Benhamou (2013), L'irrésistible ascension de l'internet, Cahiers français, janvier-février $2013 \mathrm{~N}^{\circ} 372$.

41. Freeman E.R (1984) "Strategic Management: A Stakeholder Approach", Pitman, Boston, 1984, pp-46.

42. Frooman, J. (1999), « Stakeholder Influence Strategies », Academy of Management Review, vol. 24, $\mathrm{n}^{\circ}$ 2, pp-202,.

43. Garonne C et Weygand F. (2007), Modèles d'affaires composites des start-ups TIC Problématique et typologie. Entrepreneuriat : nouveaux enjeux, nouveaux comportements, Jun 2007, Paris France, http : //www.entrepreneur-conf-paris.com/index.html, 2007

44. Gensollen M. (1999), La création de valeur sur Internet. In: Réseaux, volume $17, \mathrm{n}^{\circ} 97,1999$. Internet, un nouveau mode de communication? pp. 15-76

45. Gilder G. (1993), Metcalf's Law and Legacy, Forbes ASAP, September 1,

46. Hartmann P-M et al. (2014) Big Data for Big Business? A Taxonomy of Data-driven Business Models used by Start-up Firms Cambridge Service Alliance 2014 
47. Holmes, (2001), «Quelles alternatives au mouvement d'enclosures de la propriété intellectuelle ? », Réseaux 2001/6 (no 110), pp-80]

48. Huws U. (2003) "The Making of a Cybertariat : Virtual Work in a Real World". New York, Monthly Review Press. pp-7

49. Komorowski M. (2009), http://www.mkomo.com/cost-per-gigabyte

50. Kücklich, J. (2005) « Precarious Playbour : Modders and the Digital Games Industry», The Fibre culture Journal, 5(1)

51. Lacroix M. et Zambon S. (2002), « Capital intellectuel et création de valeur : une lecture conceptuelle des pratiques française et italienne ", Comptabilité - Contrôle -Audit 2002/3 (Tome 8), pp-66.

52. Levallois-Barth C. (2014), «L'utilisation raisonnée des données personnelles », LA JAUNE ET LA ROUGE, MARS 2014, pp-22

53. Lire M.J., et al. (2011). Big data: The Next Frontier for Innovation, Competition, and Productivity. Washington DC: McKinsey Global Institute.

54. Lordon F. (2000), " la création de valeur » comme rhétorique et comme pratique », in L'année de la régulation, Vol.4, CEPREMAP, Paris,

55. Lordon F. (2000), « la création de valeur » comme rhétorique et comme pratique », in L'année de la régulation, Vol.4, CEPREMAP, Paris,

56. Lorino $\mathrm{P}$. (1998), « Valeur pour l'actionnaire : une mode à risques », Alternatives Economiques, Septembre

57. M. Metcalfe R. et R. Boggs D. (1980), Ethernet: Distributed Packet Switching for Local Computer Networks, CSL·75.7 May 1975, reprinted February 1980

58. Martinet, A.-C., (1984) « Management stratégique : organisation et politique. » Paris: Mc Graw Hill.

59. Masahiko A. (1984), "The Co-operative Game Theory of the Firm", Oxford University Press

60. Mavrinac S. et Siesfeld T. (1998), "Measuring Intangible Investment Measures that matter: an exploratory investigation of investors' information needs and value priorites", Rapport pour l'OCDE.

61. McKelvey, M. (2001) "The Economic Dynamics of Software: Three Competing Business Models Exemplified through Microsoft, Netscape and Linux, in Innovation in the Telecommunication Industry," Economics of Innovation and New Technology, 11: 127164.

62. Mercier S. (2001), « L’apport de la théorie des parties prenantes au management stratégique : une synthèse de la littérature », Xième Conférence de l'Association Internationale de Management Stratégique 13-14-15 juin 2001 
63. Mitchell R. K., \& al. (1997), "Toward a Theory of Stakeholder Identification and Salience: Defining the Principle of Who and What Really Counts", The Academy of Management Review, Vol. 22, No. 4 (Oct., 1997), pp. 865 et suivants

64. Moulier Boutang Y. (2007) « LE CAPITALISME COGNITIF - La Nouvelle Grande Transformation », Editions Amsterdam, Paris.

65. Nagle, T. \& Sammon, D. (2014) Big Data: A Framework for Research. In: Phillips-Wren, G., Carlsson, A. \& Respicio, A. (eds.) DSS 2.0 - Supporting Decision Making With New Technologies. Amsterdam: IOS Press, 395-400.

66. OCDE (2017), RELEVER LES DÉFIS FISCAUX POSÉS PAR L'ÉCONOMIE NUMÉRIQUE.

67. Odlyzko A. et Tilly B. (2005), A refutation of Metcalfe's Law and a better estimate for the value of networks and network interconnections, Digital Technology Center, University of Minnesota, version, March 2, 2005, http://www.dtc.umn.edu/ odlyzko/doc/metcalfe.pdf

68. Osterwalder A, Pigneur Y (2010). Business Model Generation - A Handbook for Visionaries, Game Changers and Challengers. John Wiley and Sons, Inc., Hoboken, New Jersey.

69. Ouakrat A. (2012), Le concept des modèles d'affaires : éléments de définition et état de l'art : Entreprises culturelles et internet : Contenus numériques et modèles d'affaires innovants. Etat de l'art concernant la notion de modèles d'affaires de l'économie numérique liée aux entrepris.2012, pp.111-117.

70. Parmentier G. (2015) Comment construire un business model multiface ? Proposition de communication pour la $6^{\mathrm{e}}$ Rencontre du Groupe de Recherche Thématique « Innovation » de l'AIMS Septembre 2015

71. Pasquinelli M. traduit par Blanchard A. (2013), « Digitalisme. L'impasse de la media culture », Multitudes 2013/3 ( $\left.\mathrm{n}^{\circ} 54\right)$, pp- 189.

72. Penard T. (2003), Economie des réseaux et services en réseaux : une application aux stratégies concurrentielles dans l'économie numérique, chapitre « Stratégies et concurrence dans la Net-Economie ", in M. Basle et Pénard T. (Eds.) eEurope : la société européenne de l'information en 2010, Economica, 13-50, 2002

73. Perret, V. et M. Séville. (2003). Fondements épistémologiques de la recherche, in Thiétart, $\mathrm{R}-\mathrm{A}$ et coll. Méthodes de recherche en management, 2eédition. Paris : Dunod, pp-19

74. Pesqueux Y. (2002), «Organisations : modèles et représentations », GESTION, PUF pp-118.

75. Pican X. (2015) L'impact du phénomène Big Data sur les entreprises : de la gestion à la valorisation des données numériques 
gigantesques in «La propriété intellectuelle \& la transformation numérique de l'économie » INPI - Regards d'experts, pp-14

76. Pican Xavier, (2015) L’impact du phénomène Big Data sur les entreprises : de la gestion à la valorisation des données numériques gigantesques in « La propriété intellectuelle \& la transformation numérique de l'économie » INPI - Regards d'experts, pp-12

77. Pierre Collin. \& Nicolas Colin (2013). Idb. pp-80

78. Porter M. (1985), Competitive advantage. Creating and sustaining superior performance. Collier Macmillan,New York, London.

79. Porter M. E. et Kramer M R. (2011) « The Big Idea, creating shared value », Harvard Business Review, January-February, p. 62-77

80. Porter Michael E. et Kramer Mark R. (2006), « Strategy and Society: The Link between Competitive Advantage and Corporate Social Responsibility ", Harvard Business Review, December, p. 78-92

81. Propos rapporté par Pénard Thierry, Rallet Alain (2014), « De l'économie des réseaux aux services en réseaux. Nouveau paradigme, nouvelles orientations », Réseaux 2014/2 ( $\left.\mathrm{n}^{\circ} 184-185\right)$, pp-76

82. Rocheland F. et Cecere G. (2012), Modèle d'affaires numériques, données personnelles et sites web, Une évaluation empirique, Revue française de gestion - $\mathrm{N}^{\circ}$ 224/2012

83. Rouvroy A. (2017), «Des données et des Hommes. Droits et Libertés fondamentaux dans un monde de données massives ", Rapport à destination du Comité Consultatif de la Convention pour la protection des personnes au regard du traitement automatisé de données personnelles du Conseil de l'Europe. TEXTE AUGMENTE, pp-12.

84. Rouvroy A. (2017), Ibd. pp-6, reprend les propos de Delort P. (2015), « Le Big Data, » PUF, coll. Que sais-je",

85. Schmidt E., CEO de Google de 2001 à 2011, https:/googlesystem.blogspot.com/2010/08/eric-schmidt-on-futureof-search.html\#gsc.tab=0

86. Schroeck, M., and al. (2012), "Analytics: The real-world use of big data. How innovative enterprises extract value from uncertain data", IBM Institute for Business Value, Saïd Business School at the University of Oxford. 1998, pp. 55.

87. Schumpeter J. (1954 - PM) parle à ce propos d'une «Révolution de la théorie de la valeur» (History Of Economic Analysis, tome III, p. 220 et suiv.) Taylor \& Francis e-Library, 2006.

88. Simon C. (2000), «Valeur et comptabilité » Encyclopédie de comptabilité, de contrôle de gestion et audit, sous la direction de Colasse B., Economica, 2000, pp. 1245-1257 
89. Sonka S. (2016), Big Data Characteristics, IFAMA - Review, Special Issue - Volume 19 - Issue A, 2016

90. Styliani, N. (2017), Tax consequences of the digitalized economy, Committee of Experts on International Cooperation in Tax Matters, Fifteenth session, Geneva,

91. Usunier, J. C., Easterby-Smith, M., \& Thorpe, R. (1993). «Introduction to management research» Paris: Economica. 1ère edition

92. Vatteville E. (2008), «La création de valeur : de l'exclusivité actionnariale à la diversité partenariale? », Management \& Avenir, Vol. 4, $\mathrm{N}^{\circ} 18$, pp-90 et 91

93. Verstraete T. al, (2012) « Le business model : une théorie pour des pratiques », Entreprendre \& Innover, 2012/1 n 13, pp. 7-26.

94. Verstraete T. et Jouison-Lafitte E. (2009), business model pour entreprendre - Le modèle GRP : théorie et pratique, Collection De Boeck,

95. Verstraete T. et Jouison-Lafitte E., (2007), «Trois théories pour conceptualiser la notion de business model en contexte de création d'entreprise ». XVI conférence internationale de management stratégique. pp-4

96. Walker R. (2015), From Big Data to Big Profits, SUCCESS WITH DATA AND ANALYTICS, $\mathrm{p}$ : xxi Oxford University Press

97. Weinberger D. (2011), "Too big to know: Rethinking know ledge now that the facts aren't the facts, experts are everywhere, and the smartest person in the room is the room". New York, NY: Basic Books

98. Worst J. (2014), The case of the "Big Data" revolution, Working Paper Maastricht School of Management No. 2014/34

99. Xu H., et Zhang J. (2014), "Clickstream Big Data and "Delivery before Order Making", Colloquium 2014, School of Management, Huazhong University of Science \&Technology 